\title{
Impact of crisis resolution and home treatment services on user experience and admission to psychiatric hospital
}

\author{
Victoria Barker, ${ }_{1}^{1}$ Mark Taylor, ${ }^{2}$ Ihsan Kader, ${ }^{2}$ Kathleen Stewart, ${ }^{2}$ Pete Le Fevre ${ }^{1}$
}

The Psychiatrist (2011), 35, 106-110, doi: 10.1192/pb.bp.110.031344

${ }^{1}$ Royal Edinburgh Hospital, Edinburgh; ${ }^{2}$ Intensive Home Treatment Team, Edinburgh

Correspondence to Mark Taylor (marktaylor2@nhs.net)

First received 31 May 2010, final revision 30 Aug 2010, accepted 5 Nov 2010

\begin{abstract}
Aims and method Crisis resolution and home treatment (CRHT) teams began operating in Edinburgh in late 2008. We ascertained service users' and carers' experiences of CRHT using a standardised questionnaire. We also assessed the impact of CRHT on psychiatric admissions and readmissions by analysing routinely collected data from November 2003 to November 2009.
\end{abstract}

Results There was a $24 \%$ decrease in acute psychiatric admissions in the year after CRHT began operating, whereas the previous 5 years saw an $8 \%$ reduction in the admission rate. The mean duration of in-patient stay fell by 6.5 days ( $22 \%$ decrease) in the 12 months following CRHT introduction, alongside a $4 \%$ decrease in readmissions and a 17\% reduction in Mental Health Act 1983 admissions. Although the mean response rate was low (29\%), $93 \%$ of patients reported clinical improvement during $\mathrm{CRHT}$ care, $27 \%$ of patients felt totally recovered at discharge from CRHT, $90 \%$ of patients felt safe during CRHT treatment, and $94 \%$ of carers said their friend or relative got better with CRHT input.

Clinical implications Crisis resolution and home treatment service in Edinburgh had a positive impact during the first 12 months in terms of reduced admissions, reduced duration of in-patient stay and reduced use of the Mental Health Act. The service can catalyse a more efficient use of in-patient care. Service users and carers report high rates of improvement and satisfaction with CRHT.

Declaration of interest M.T., I.K., and K.S. work in a CRHT.
The Department of Health in England and Wales has recommended crisis resolution and home treatment (CRHT) in its best practice and policy implementation guides since $2002 .^{1-3}$ In 2007 it described CRHT as a key step in implementing the mental health National Service Framework, partly to ensure in-patient care is used only where necessary. ${ }^{4}$ In 2005, Johnson et $a l^{5}$ undertook a randomised controlled trial of CRHT and found that 8 weeks after the introduction of CRHT, admission rates in the general adult psychiatry population were reduced from 59 to $22 \%$. Reduced admission rates have been demonstrated by other studies, including a large observational study of 229 of 303 teams in England. ${ }^{6,7} \mathrm{~A}$ number of investigations have also showed reduced length of in-patient stay following introduction of CRHT. ${ }^{8-10}$

Crisis resolution and home treatment teams have now been implemented throughout England and Wales. Jethwa et $a l^{7}$ performed an evaluation of the long-term effects of the introduction of CRHT in Leeds and demonstrated a significant reduction in admission rates of $37.5 \%$ in the first year. In contrast, a study by Tyrer et $a l^{11}$ found only a $7.7 \%$ reduction in admissions and no significant reduction in length of in-patient stay after the introduction of CRHT in Cardiff.

Services in Scotland were not constrained by the Department of Health national service framework and did not incorporate functionalised teams such as assertive outreach, early intervention and CRHT teams into clinical services until relatively recently. A trial of CRHT in Falkirk in 2006 reported reduced in-patient admissions and length of stay, and demonstrated positive feedback from service users. $^{12,13}$

In late 2008, CRHT was introduced in Edinburgh. We wanted to evaluate the impact on admission rates and length of stay following the introduction of CRHT. Additionally, we examined readmission rates (which previous studies have not investigated), rates of compulsory admission, and patients' and carers' satisfaction with and experience of the CRHT.

\section{Method}

In November 2008, two high-fidelity CRHT teams with dedicated medical input and managerially supported 
gatekeeping roles were introduced in Edinburgh for the care of adults, aged 18-65 years who had mental health problems and who posed a significant risk to themselves or others. The teams operated in combination with a nurse-led emergency assessment service for self-referrals. The aims of these teams were to provide a community-based alternative to in-patient psychiatric care, to facilitate early discharge from hospital and to reduce hospital readmission rates.

Edinburgh mental health services previously consisted of a traditional combination of in-patient and community mental health services, but no functionalised teams. Edinburgh has a total resident population of 471650 (the seventh most populous city in Britain). The city's demographics led to two CRHT teams being instituted, one for north Edinburgh and one for south Edinburgh. Each team consisted of 1 consultant psychiatrist, 1 staff grade psychiatrist (2 days/week), 1 nurse team leader, 13 band $5 / 6$ nurses, 1 social worker, 1 occupational therapist and 1 support worker. A nurse consultant covered both CRHT teams. Edinburgh CRHT provides an around-the-clock service accepting referrals from within mental health services and from other sources, including general practitioners, accident and emergency departments, the police and the voluntary sector (but not self-referrals). The CRHT teams had full gatekeeping over admissions to the acute general adult psychiatry wards. Patients with a wide variety of diagnoses, using ICD-10 operational criteria and coding, ${ }^{14}$ were assessed and managed by the CRHT teams (Fig. 1). The mean treatment duration for patients taken on was 3 weeks.

A retrospective examination of collated data on admissions to acute general adult psychiatric wards for the 5 years before, and the 1 year following, the introduction of CRHT was made. The period analysed was from 15 November to 14 November for the years 2003-2004, 20042005, 2005-2006, 2006-2007, 2007-2008 and 2008-2009. Demographic information on patients admitted in this period was analysed by gender and diagnosis at the time of discharge. Data examined included:

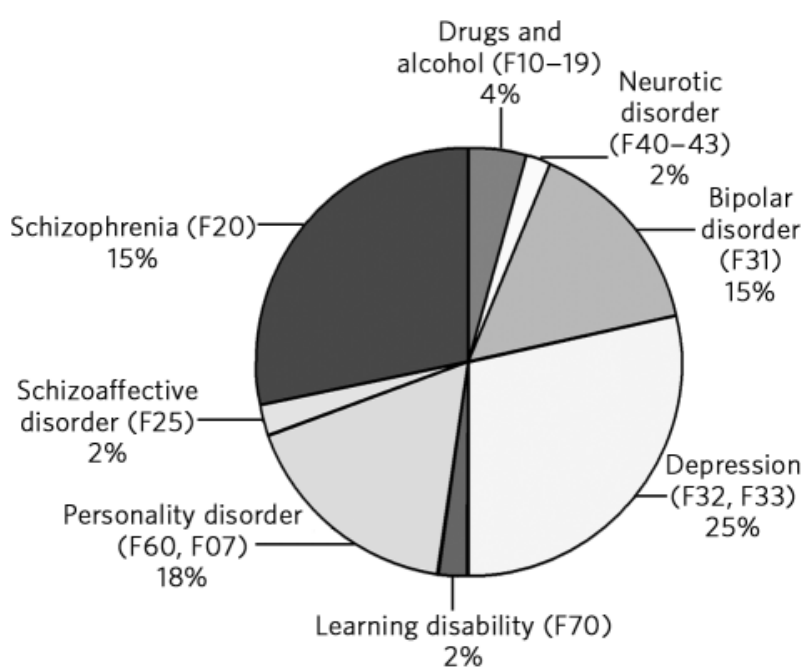

Fig 1 Diagnostic breakdown of patients assessed and managed by the two Edinburgh CRHT teams from November 2008 to November 2009.
- number of admissions;

- length of in-patient stay;

- readmission rates according to the Scottish government target (readmission to a psychiatric hospital for at least a further continuous 7 days within 365 days of discharge from the original admission);

- number of patients admitted under the Mental Health (Care and Treatment) (Scotland) Act 2003.

Descriptive analysis was applied to the data. Unpaired $t$-tests were performed on the admission, readmission and length-of-stay data. The last were transformed before parametric analysis, because in-patient stay has been established as being non-normally distributed. The $\chi^{2}$-test was performed on the data on detention under the Mental Health Act 1983.

The second part of the study evaluated patients' and carers' experiences of and satisfaction with CRHT. As part of the routine discharge process from CRHT, all service users and their carers with whom the CRHT teams worked were posted an anonymised satisfaction feedback questionnaire for completion after the treatment episode. The questionnaires assessed quantitative and qualitative patient and carer outcomes and are essentially identical to the shortform version of the Patient Satisfaction Questionnaire (PSQ-18). The original PSQ consisted of 80 items; the PSQ-18 is a short version that retains many characteristics of its full-length counterpart. The PSQ subscales have been demonstrated to show acceptable internal consistency, construct validity and reliability. The PSQ-18 is appropriate for use in situations where the need for brevity precludes administration of the full-length PSQ and has been used extensively in primary care settings. ${ }^{15,16}$ A postage-paid return envelope was provided to facilitate return of the questionnaires. Data were then collated and analysed.

\section{Results}

There were two distinct elements to the study. First, there was an examination of the impact of CRHT introduction on in-patient services. The mean number of admissions per annum for the 5 years before CRHT introduction was 1266 (647 males, 619 females), with an $8 \%$ fall in admission rate observed in the period November 2003 to November 2008. The number of admissions for the year following CRHT was 955 (485 male, 470 female). This represented a reduction of $24.6 \%$ from the annual mean for the previous 5 years (unpaired $t$-test, $P<0.0001$ ) (Fig. 2). This compares to reductions from 2007-2008 to 2008-2009 in the four areas of Scotland that have not introduced CRHTs (Tayside, $6.8 \%$; Grampian, 9.0\%; Highland, 9.4\%; Fife, 14.1\%) (details available from M.T. on request).

The length of stay was reduced by 9.21 days $(28.58 \%)$, from a mean length of stay of 32.23 days from November 2004 to November 2008 to 23.02 days in November 2008 to 2009 (Fig. 3). As in-patient stay has been established as being non-normally distributed, the data were transformed and subsequent unpaired $t$-tests showed a significant difference $(P<0.0001)$ in mean length of stay. Mean length of stay for the year preceding CRHT introduction from November 2007 to November 2008 was 29.5 days. 


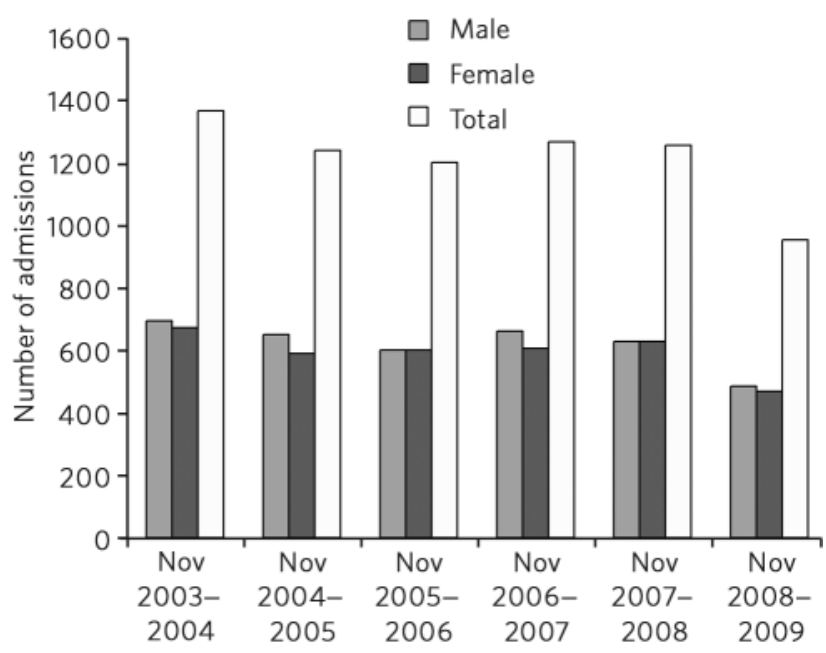

Fig 2 Number of admissions to acute general adult psychiatry wards in Edinburgh in the 5 years before, and 1 year following, CRHT introduction. There is a significant decrease in admissions following CRHT introduction.

There was a reduction of 6.48 days with CRHT introduction $(P=0.076)$. The mean readmission rate according to the readmission target for the 5 years before CRHT introduction was 348 compared with 282 following introduction. This was found to be non-significant (4\% reduction, $P=0.152)$ when using the unpaired $t$-test.

Using the absolute numbers, in the year following CRHT introduction there was a $17 \%$ reduction in patients detained under the Mental Health Act on admission compared with the 12 months before CRHT introduction, but this was found not to be statistically significant $\left(P=0.335, \chi^{2}=0.93\right.$, d.f. $\left.=1\right)$ using $\chi^{2}$ with Yates' correction. There was also a $25 \%$ reduction in the number of patients admitted informally in the year following CRHT introduction, reflecting the overall reduction in admissions.

The second part of the study related to service user and carer satisfaction with the service. A total of 560 patients were taken on by the CRHT teams in the year from November 2008 to November 2009. These patients (and their carers where appropriate) were provided with feedback questionnaires on discharge. There was a mean response rate of $29 \%$ for the feedback questionnaires from the two CRHT teams (175 replies): $93 \%$ of patient respondents reported improved mental health during CRHT involvement and $31 \%$ felt totally recovered at discharge from CRHT; $94 \%$ of carers that responded said their friend or relative got better with CRHT input; $90 \%$ of responding service users and carers felt they were involved in decisions about care and treatment; $89 \%$ of service users felt safe during CRHT involvement, and $78 \%$ felt prepared to move on at the time of discharge from CRHT.

Many freestyle comments were also received. An example from a service user is given here: 'Although I find it very difficult to trust people I do not know and allow them into my house, I found all staff who visited aware of this and they were very good at helping me feel safe with them. My feelings and thoughts were listened to and heard and treated as real and important and never dismissed. The

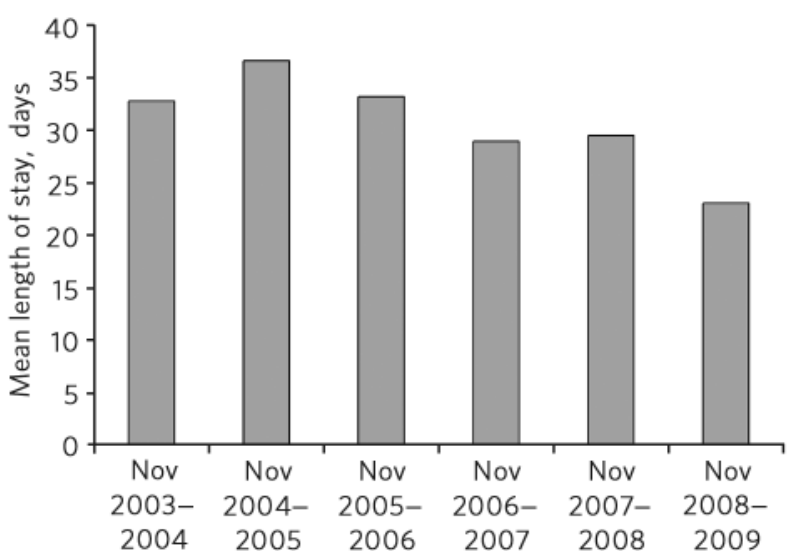

Fig 3 Mean length of in-patient stay in acute general adult psychiatry wards in Edinburgh in the 5 years before, and 1 year following, $\mathrm{CRHT}$ introduction. There is a significant reduction in length of stay with the introduction of CRHT.

encouragement was always there but I never felt pushed to go at a faster pace than I was able. Having regular contact with medical staff involved me in all decisions around my care and changes in medication. Had I not had input from intensive home treatment team [our team name] I would have been admitted to hospital, which would have set my recovery much further back and increased my feelings of being unable to manage my mental health.'

\section{Discussion}

\section{Principal findings}

The introduction of high-fidelity CRHT teams in Edinburgh reduced the number of admissions to in-patient psychiatric wards. For those patients admitted, the length of stay was significantly reduced, but this did not have a detrimental impact on readmission rates, which remained stable. There was a reduction in the number of patients admitted under the Mental Health Act, but this was not statistically significant. Service users and carers reported high rates of recovery after and satisfaction with CRHT intervention.

\section{Context}

Our findings provide evidence to support most previous studies, ${ }^{5-10,12,13}$ which demonstrated that CRHT teams reduce admission rates and reduce length of in-patient stay. Tyrer et $a l^{11}$ found a non-significant reduction of $7.7 \%$ in admissions following the introduction of a CRHT in Cardiff, but they examined a control population in which they found the same reduction of $7.7 \%$ in admissions, which they acknowledge consisted of a population so dissimilar in size and socioeconomic circumstances that it was effectively incomparable with the test group.

We demonstrated a $17 \%$ reduction in admissions under the Mental Health Act and a 25\% reduction in informal admissions in the year following CRHT introduction, compared with the previous 12 months. This is substantially greater than reductions found in areas of Scotland where CRHT has not been introduced (details available 
from M.T. on request). Indeed, the reductions in areas without CRHTs are similar to those found in the 5-year period before the introduction of CRHTs in Edinburgh. Our findings reflect an overall reduction in admissions and are in stark contrast to the finding by Tyrer et al that introduction of CRHT reduced informal admissions but was accompanied by increased numbers of compulsory admission. ${ }^{\text {ll }}$ Our results replicated the finding of Johnson et al that there was a reduction in compulsory admissions with the introduction of CRHT but that this was not significant. ${ }^{8}$ Tyrer et al hypothesised that the work of CRHT teams maintains patients in the community, preventing informal admissions, but results in some patients' condition deteriorating to such a point that compulsory admission is eventually necessary. ${ }^{11}$ Our results contradict this hypothesis and reflect the fact that crisis care in the community maintains patients in a stable condition without requiring later compulsory hospital admission. This is also reflected by our demonstration that readmission rates were not increased following the introduction of CRHT, which included early discharge management and subsequent crisis intervention for those discharged from in-patient care.

The Tyrer et al study was performed on a relatively small and specific city-centre population, which may contain more complex patients who are difficult to manage, come from a poorer socioeconomic background and have fewer resources. ${ }^{11}$ In contrast to this, our CRHT teams covered a wide and socially diverse geographic area, which may account for some of the differences in findings and may indicate that our results are more generalisable to services in other, larger catchment areas.

Differing patterns of staffing and operational policy also led to different outcomes in service. Evidence from the National Audit Office demonstrates that where CRHT teams are sufficiently staffed and resourced they reduce in-patient admissions. ${ }^{17}$ The success of CRHT services appears to be dependent to some extent on there being medical support and full gatekeeping responsibility intrinsic to the team. ${ }^{18}$ The results demonstrated by Tyrer et al may reflect failures in effective implementation of the CRHT model, including these essential elements. It has been found that if there are limited resources or if essential functions such as gatekeeping are absent, then services seem to deviate from the core aims of CRHT service provision and spend more time performing assessments and providing longer-term care. ${ }^{17}$ The Edinburgh CRHT teams had both medical support and full gatekeeping responsibility, and thus effective implementation of the CRHT model may have been key to the success in reducing admissions and duration of stay.

A number of factors have been proposed to act as facilitators or barriers to determine whether national mental health policies such as the introduction of CRHT proceed to implementation and long-term application. These include initial availability of resources, training, presence of opinion leaders and champions, consistency of local policy and practice guidance, systems for assessing practice fidelity, effective feedback systems to staff, staff turnover and continuing staff training. It has been argued that the greatest impact on public health comes not from the adoption or early implementation of evidence-based practice but from reduced levels of unjustified variability in clinical practice. ${ }^{19}$ Although there are now many published clinical guidelines, there are relatively few studies on how to put guidelines into cost-effective routine practice. This makes consistent and effective implementation of evidencebased interventions such as CRHT in different areas extremely difficult, and variability in implementation may account for differences in outcomes seen between this study and that of Tyrer et al. There is, as yet, no validated system of assessing and comparing practice fidelity across studies or regions.

Our results evaluating patients' and carers' attitudes to the CRHT teams in which there were high satisfaction rates suggest that the Edinburgh teams' practice was high in quality and good therapeutic relationships were developed with both service users and carers. This did, however, reflect the attitudes of only about $30 \%$ of the total service user group and may not account for more negative service user experiences that were not reported. Our results of reduced admission rates and length of stay are consistent with the findings of the National Audit Office ${ }^{17}$ and reflect highfidelity practice.

\section{Limitations}

As our study was a retrospective rather than a prospective examination, it could not be compared with a control population. Confounding factors such as changes in the economic climate and in other mental health and social service provisions during this period may have affected the outcomes and were not considered in this study. It should, however, be noted that there were no changes in local mental health services at this time (e.g. early intervention service or crisis houses), other than the introduction of the CRHT and associated reduction in acute general adult in-patient beds, which might account for the reduction in admissions.

The results of this study must be interpreted in the context of the remodelling of the service that occurred at the time of CRHT introduction and involved an approximately $30 \%$ planned closure in acute general adult psychiatry beds. It is, therefore, difficult to know whether the admission rates were reduced as a consequence of there being fewer beds available into which to admit patients. This is a common problem in assessing the impact of CRHT, as service development generally anticipates the introduction of CRHT teams by reducing in-patient bed numbers. The reduction in beds may have increased pressure on in-patient wards to discharge patients earlier than would previously have been the norm, resulting in a reduction in length of stay. The fact that there was no significant increase in readmission rates suggests that if this did occur, then it did not have a negative impact on patient outcome; this is corroborated by the high satisfaction rates on feedback from service users. This may be due to the involvement of CRHT in facilitating early discharge and supporting these patients in the community following discharge.

A second consequence of reduced bed numbers could be fewer admissions at initial presentation, with a consequent increase in admissions of patients who have become increasingly unwell as reflected by subsequent admission compulsorily under the Mental Health Act. This study suggests that in Edinburgh this was not the case. This 
may reflect provision of care to patients in the community by the CRHT teams as an alternative to admission.

Patients and carers who provided feedback on the service positively evaluated their experience of CRHT. As the mean response rate was only $29 \%$, however, it is difficult to generalise this to the other $71 \%$ of service users and carers who did not respond. It may be that this resulted in a bias towards those patients who did well with the service responding and those who had a less successful experience with CRHT and remained more unwell, being less likely to return the feedback questionnaire. We specifically decided against enhancing the return rate by requesting completion at face-to-face discharge, as this may have introduced bias.

There is a need to further examine the effect of specific variations in staffing and practice of CRHT teams on outcomes. Ideally, a further randomised controlled trial of CRHT teams should be performed, but this becomes increasingly difficult as greater numbers of mental health services have existing CRHT teams and therefore recruiting and instituting appropriate control groups and interventions is a difficult prospect. Crisis resolution and home treatment teams vary greatly in their aims and in the way they are configured. This study supports the highfidelity model of CRHT and demonstrates that it can have consistent positive outcomes and can catalyse a more efficient use of in-patient care. It also reports good levels of user satisfaction and user-rated outcome in those that returned feedback. It thus lends weight to the increasing trend towards community-based crisis care throughout the UK.

\section{About the authors}

Victoria Barker is a trainee psychiatrist at the Royal Edinburgh Hospital, Edinburgh. MarkTaylor and Ihsan Kader are consultant psychiatrists, and Kathleen Stewart is a nurse consultant, all in the Intensive Home Treatment Team, Edinburgh. Pete Le Fevre is a consultant psychiatrist at the Royal Edinburgh Hospital, Edinburgh.

\section{References}

1 Department of Health. Fast-Forwarding Primary Care Mental Health: Gateway Workers. Department of Health, 2002.

2 Department of Health. Mental Health Policy Implementation Guide: Community Mental Health Teams. Department of Health, 2002.
3 Department of Health. Fast-Forwarding Primary Care Mental Health: Best Practice Guidance. Department of Health, 2003.

4 National Institute for Mental Health in England, Care Services Improvement Partnership. Guidance Statement on Fidelity and Best Practice for Crisis Services. Department of Health, 2007.

5 Johnson S, Nolan F, Pilling S, Sandor A, Hoult J, McKenzie N, et al. Randomised controlled trial of acute mental health care by a crisis resolution team: the north Islington crisis study. BMJ 2005; 331: 599604.

6 Glover G, Arts G, Babu KS. Crisis resolution/home treatment teams and psychiatric admission rates in England. Br J Psychiatry 2006; 189: 441-5.

7 Jethwa K, Galappathie N, Hewson P. Effects of crisis resolution and home treatment team on in-patient admissions. Psychiatr Bull 2007; 31: 170-2.

8 Johnson S, Nolan F, Hoult J, White IR, Bebbington P, Sandor A, et al. Outcomes of crises before and after introduction of a crisis resolution team. Br J Psychiatry 2005; 187: 68-75.

9 Joy C, Adams C, Rice K. Crisis intervention for people with severe mental illness. Cochrane Library, 2002; issue 2. Update Software.

10 Catty J, Burns T, Knapp M, Watt H, Wright C, Henderson J, et al. Home treatment for mental health problems: a systematic review. Psychol Med 2002; 32: 383-401.

11 Tyrer P, Gordon F, Nourmand S, Lawrence M, Curran C, Southgate D, et al. Controlled comparison of two crisis resolution and home treatment teams. Psychiatrist 2010; 34: 50-4.

12 Coutts P, McLaren G, Crawford C. Providing alternatives to inpatient care: the intensive home treatment team pilot in Forth Valley. Ment Health Rev 2006; 11: 37-40.

13 Jackson C. Home but not alone. Ment Health Today 2007; Nov: 16-7.

14 World Health Organization. The ICD-10 Classification of Mental and Behavioural Disorders. Clinical Descriptions and Diagnostic Guidelines. WHO, 1992.

15 Marshall GN, Hays RD. The Patient Satisfaction Questionnaire Short Form (PSQ-18). RAND, 1994

16 Grogan S, Conner M, Norman P, Willits D, Porter I. Validation of a questionnaire measuring patient satisfaction with general practitioner services. Qual Health Care 2000; 9: 210-5.

17 National Audit Office. Helping People through Mental Health Crisis: The Role of Crisis Resolution and Home Treatment Services. National Audit Office, 2008 (http://www.nao.org.uk/publications/0708/helping_ people_through_mental.aspx).

18 Johnson S, Needle J, Bindman J, Thornicroft G. Crisis Resolution and Home Treatment in Mental Health. Cambridge University Press, 2008.

19 Tansella M, Thornicroft G. Implementation science: understanding the translation of evidence into practice. Br J Psychiatry 2009; 195: 283-5. 